\title{
The Influence of Psychological Distance on Ambiguity Decision Making: A Perspective Based on the Construal Level Theory
}

\author{
Zeyu Tan, Yaozhong Liu \\ School of Management, Jinan University, Guangzhou, China \\ Email:hntanzeyu@163.com
}

How to cite this paper: Tan, Z. Y., \& Liu, Y. Z. (2018). The Influence of Psychological Distance on Ambiguity Decision Making: A Perspective Based on the Construal Level Theory. Psychology, 9, 997-1004. https://doi.org/10.4236/psych.2018.95063

Received: April 12, 2018

Accepted: May 11, 2018

Published: May 17, 2018

Copyright (c) 2018 by authors and Scientific Research Publishing Inc. This work is licensed under the Creative Commons Attribution International License (CC BY 4.0).

http://creativecommons.org/licenses/by/4.0/

\begin{abstract}
Based on the construal level theory, this paper investigates the influence of different dimensions of psychological distance on the ambiguity decision making. Based on the four dimensions of psychological distance, this paper uses single factor within-subjects experiment design and randomly assigns subjects into each dimension to participate in the experimental manipulation so as to test whether different psychological distance dimensions have an influence on the ambiguity decision making. The results show that, under the conditions of time distance, space distance, social distance and probability, the closer the psychological distance is to decision making, the lower the construal level for the decision event or the object, thereby more inclined to ambiguity avoidance. Thus, the closer the psychological distance of decision event is, the higher degree of the individual's ambiguity avoidance.
\end{abstract}

\section{Keywords}

Psychological Distance, Construal Level, Ambiguity Avoidance

\section{Introduction}

We all have to face all kinds of decision-making situations in our daily life. In some decision situations, the probability of the occurrence of a specific event is known, such as throwing a sieve or tossing a coin, and the decision made in the case is actually a kind of risk type decision. In contrast, the probability of the occurrence of a specific event in some decision-making situations is uncertain, such as investment decision, choice of medical plan and so on. This kind of decision-making in the situation is called ambiguity decision in which the probability of the event occurrence is partly uncertain or completely uncertain. 
Ellsberg (1961), via bottle-choosing task, found that when people face both risk type options and ambiguity options, they are more likely to choose the risk type options; this phenomenon is called ambiguity avoidance. The researchers also found ambiguity avoidance phenomena are frequently shown in the context of insurance decisions and medical decisions (Camerer, Colin, Weber, \& Martin, 1991; Fox \& Weber, 2002). However, some researches showed that people are not always averse to ambiguity in ambiguity decision-making, their degree of ambiguity avoidance will be affected by other factors such as capacity, gender, profit and loss framework, which may lead to the weakening of ambiguity avoidance, and even lead to ambiguity preference inversion, i.e. ambiguity seeking. Heath and Tversky (1991) believed that the degree of ambiguity avoidance is affected by the size of the capacity: if people feel capable or have sufficient knowledge of uncertain sources, the ambiguity avoidance phenomenon will be weaken. Pulford (2009) found that the optimistic personality trait could regulate the ambiguity aversion effect in subjects, and subjects with optimism in the decision-making prospect are apt to seek ambiguity. In addition, researchers have found that people like to evade the ambiguity in the profit framework, but like to seek ambiguity in the loss framework (Osmont, Cassotti, Agogué, Houdé, \& Moutier, 2015).

Most of these factors are discussed from the point of view of decision makers or decision-making tasks, and the factors that affect decision making are influenced by different perceptions of psychological distance in decision situations besides decision makers or decision-making tasks. More specifically, they can be divided into making decisions for themselves and for strangers, making decisions for the present and for the future, for the event with a very large probability of occurrence and for the event with a small probability of occurrence, for the event with a short space distance and the event with a long space distance. In these decision-making situations, individuals will perceive the distances differently based on the self-others, present-future, large probability - small probability, and short space distance-long space distance, such distance perceived by the reference point of self-immediate experience are actually the psychological distance (Trope \& Liberman, 2010). In the past, the influence of psychological distance on decision making was mainly focused on the fields of risk type decision, ethical decision and so on (Gong, Iliev, \& Sachdeva, 2012, Chen \& He, 2014), and few studies were carried out to explore the influence of psychological distance on ambiguity decision making. Therefore, the application of psychological distance in ambiguity decision making field is a research direction that can be fully expanded.

Psychological distance refers to self-orientation, the distance between the time and space, the closeness between the social individuals and the probability of occurrence. It includes four dimensions: time distance, space distance, social distance and probability, in which the time distance is based on "the moment" as the starting point, about the perception of the temporal distance from the event 
occurrence; the space distance is based on "this place" as the starting point for the perception of the spatial distance from the event occurrence; the social distance is based on the "self" as the starting point for the perception of the distance between others and the self; the probability distance is based on the "reality" as the starting point for the perception of the likelihood of event occurrence (Todorov, Goren, \& Trope, 2007; Trope \& Liberman, 2010). Construal level theory believes that (Trope \& Liberman, 2010), the psychological distance will affect the individual's representation of the event, the closer the psychological distance is, the lower the level of construal, and individuals will take concrete, complex and situational representation ways; the farther the psychological distance is, the higher the level of construal, and the individual will take abstract, simple and de-situational representation ways. The size of the psychological distance causes different construals of decision-making tasks, thereby affecting the individual preference. When individuals are in decision-making, the weight of the value associated with the low construal level will decrease with the increase of psychological distance, and the weight of the value associated with the high construal level will increase with the increase of psychological distance. Liu and Onculer (2017), from the perspective of time distance dimension and the probability dimension of psychological distance, found that psychological distance has a significant impact on ambiguity avoidance. As time distance increases or the probability becomes lower, individual's ambiguity avoidance degree decreases and is more inclined to ambiguity neutralization or ambiguity seeking. What about the influence of other dimensions of psychological distance on ambiguity decision? The construal level theory holds that the same mechanism exists in all categories of psychological distance, and there is a same self-centered reference point in different psychological distance dimensions, which is similar for the representation of decision tasks. Therefore, we think that the influence of different dimensions of psychological distance on ambiguity decision may be similar, that is, under the different dimensions of psychological distance, individuals may be more inclined to ambiguity avoidance when they perceive that the psychological distance is closer.

In conclusion, this study attempts to explore the influence of the psychological distance on the ambiguity decision by a series of situational experiments from the dimensions of time distance, space distance, social distance and probability, and makes the following hypotheses:

H1: Compared with the longer time distance, the individual is more inclined to ambiguity avoidance when the time distance is shorter;

$\mathrm{H} 2$ : Compared with the farther space distance, the individual is more inclined to ambiguity avoidance when the space distance is nearer;

H3: Compared with making decision for strangers, the individual is more inclined to ambiguity avoidance when making decision for herself/himself;

H4: Compared with the lower probability level, the individual is more inclined to ambiguity avoidance under the higher probability level. 


\section{Methods}

\subsection{Subjects and Experimental Design}

A total of 162 college students or graduate students from Guangzhou participated in this experiment. Excluding the data in which only choice 1 or 7 was chosen due to mistakes in comprehension and data that include question omissions. A total of 145 valid questionnaires were collected, including 69 males and 76 females. The average age is 21.31 years old $(S D=2.23)$.

\subsection{Experimental Materials}

The materials of this ambiguity decision-making experiment were derived from Ellsberg's classic bottle-choosing task: In a lottery game, there are two opaque bottles marked as A and B, containing two different colors of balls (red and blue). There are 100 balls in bottle A: 50 blue balls and 50 red balls. While there are also 100 balls in bottle B: the number of blue ball is within $40-60$, the number of red balls is within 60 - 40 (the proportions of red and blue balls are randomly allocated by computer). The subjects need to choose a bottle to pick a ball, if the blue ball is got, he/she will receive 500 yuan of cash reward. Otherwise, there is no reward. The choosing preferences are expressed by a 7-point scale, in which "1" represents the risk option, "7" represents the ambiguity option, and " 4 " represents the middle item. The time distance dimension requires the subjects to pick the ball from a bottle under the condition of obtaining the experimental reward 1 day (near future) or 3 months (far future) after the test; the space distance dimension requires the subjects to pick the ball from a bottle under the condition of participating in this lottery game in Guangzhou (nearer distance) or in New York (farther distance); the social distance dimension requires the subjects to pick the ball from a bottle under the condition of making decisions for themselves (shorter distance) or for strangers (longer distance); the probability dimension requires the subjects to pick the ball from a bottle under the condition of obtaining the experimental reward under a $99 \%$ chance (shorter distance) or under a $1 \%$ chance (longer distance).

\subsection{Experimental Design and Procedures}

First of all, the different dimensions of psychological distance were subject to a manipulation test, the psychological distance size perceived on dimensions of the time distance, space distance, social distance and probability was scored via a 7-point scale: "1" represents very close, "7" represents very far, "4" represents the median degree, and then the subjects were asked to fill out the choosing preference scale under different psychological distance dimensions. The different dimensions of psychological distance were subject to within-subjects design.

\section{Results}

\subsection{Manipulation Test of Psychological Distance}

A test was performed on the validity of manipulating the time distance, space 
distance, social distance and probability, the paired sample $\mathrm{T}$ test showed that there are significant differences in the selection results of time distance $\left(M_{\text {near }}\right.$ $\left.(S D)=2.44(1.40), M_{\text {far }}(S D)=5.74(1.71), t_{(33)}=-7.90, p<.01\right)$, space distance $\left(M_{\text {near }}(S D)=2.03(1.37), M_{\text {far }}(S D)=5.15(1.63), t_{(39)}=-8.17, p<.01\right)$, social distance $\left(M_{\text {near }}(S D)=3.09(1.22), M_{\text {far }}(S D)=5.71(1.06), t_{(33)}=-8.28, p<.01\right)$ and probability $\left(M_{\text {near }}(S D)=2.78(1.44), M_{\text {far }}(S D)=4.68(1.67), t_{(36)}=-4.94, p\right.$ $<.01)$, which indicates that the manipulation of psychological distance is effective.

\subsection{Ambiguity Avoidance Preference}

A total of 145 copies of valid data were collected and the data were analyzed via SPSS21.0, and the results were shown in Table 1. It can be seen that, in four kinds of psychological distance dimensions, the low level of construal and the high level of construal both showed a significant difference in the ambiguity avoidance degree. Under the time distance dimension, the degree of ambiguity avoidance of the subjects in the short time distance is significantly higher than the long time distance $\left(t_{(33)}=2.97, p<.05\right)$. Under the spatial distance dimension, the degree of ambiguity avoidance of the subjects in the short space distance is significantly higher than the long space distance $\left(t_{(39)}=2.26, p<.05\right)$. Under the social distance dimension, the degree of ambiguity avoidance of the subjects self-decision making is significantly higher than that making decisions for others $\left(t_{(33)}=2.54, p<.05\right)$. Under the probability dimension, the degree of ambiguity avoidance of the subjects in the high probability is significantly higher than those in low probability $\left(t_{(36)}=3.81, p<.01\right)$. And the hypotheses $1,2,3$ and 4 have all been confirmed, thus proving that the closer the psychological distance is, the higher the degree of ambiguity avoidance of the subjects.

\section{Discussion}

Through situational questionnaire experiments, this study explored the influence of psychological distance on ambiguity decision making from the dimensions of

Table 1. The results of t-test on ambiguity avoidance tendency under different psychological distance dimensions.

\begin{tabular}{|c|c|c|c|c|c|}
\hline Psychic distance & Variables & $N$ & $M$ & $S D$ & $t$ \\
\hline \multirow{2}{*}{ Time distance } & Short time distance & 34 & 2.97 & 1.60 & $2.97^{\star}$ \\
\hline & Long time distance & 34 & 4.38 & 1.78 & \\
\hline \multirow{2}{*}{ Space distance } & Short space distance & 40 & 3.43 & 1.55 & $2.26^{*}$ \\
\hline & Long space distance & 40 & 4.30 & 1.45 & \\
\hline \multirow{2}{*}{ Social distance } & Self-decision making & 34 & 2.76 & 1.72 & $2.54^{\star}$ \\
\hline & Making decisions for others & 34 & 3.91 & 1.60 & \\
\hline \multirow{2}{*}{ Probability } & High probability & 37 & 2.95 & 1.58 & $3.81^{\star *}$ \\
\hline & Low probability & 37 & 4.62 & 1.64 & \\
\hline
\end{tabular}

Note: ${ }^{*}$ indicates $p<.05,{ }^{* *}$ indicates $p<.01$. 
time distance, space distance, social distance and probability. The results showed that psychological distance had a significant effect on ambiguity decision making, and the degree of ambiguity avoidance increases when psychological distance gets closer. The causes of this phenomenon can be analyzed from the angles of emotion and cognition.

Previous studies have shown that (Rubaltelli, Rumiati, \& Slovic, 2010), ambiguity avoidance depends on individual emotional experience: negative emotions led to individual's ambiguity avoidance, and positive emotions led to individual's ambiguity seeking. Pulford (2009) found that optimistic personality trait could regulate the ambiguity aversion effect of the subjects, and individual with a higher level of optimism tends to seek ambiguity. Cen (2016) analyzed the psychological distance from the angles of event probability, time distance and space distance, and found that different psychological distances could cause two kinds of psychological representations with different construal levels, and thus showing different optimistic deviation effects. More positive deviations could be caused by far psychological distance, while minor positive deviations could be caused by near psychological distance. In addition, Liu and Onculer (2017) considered that far psychological distance, compared to near psychological distance, could reduce the negative emotional experience of individual and reduce the tendency of ambiguity avoidance. When individuals are confronted with events that have a long psychological distance, the formation of the high construal level could make them focus on the core characteristics of events and reduce their concern for emotional response, and the high construal level could reduce the focus on the emotional goals of decision-making, thus weakening the intensity of the potential negative emotional response and leading to a lower level of ambiguity aversion. Therefore, in the face of probability-determined and probability-ambiguity options, they are more likely to choose ambiguity options with uncertain probabilities. On the contrary, the low level of construal formed by the short psychological distance makes the individuals more concerned with the specific information and emotional goals, thus increasing the collection of information related to decision making and strengthening the role of decision-making emotional goals. Therefore, the ambiguity aversion degree is enhanced, and individuals were more inclined to ambiguity avoidance.

Furthermore, preceding experimental studies showed that (Liberman \& Trope, 1998; Todorov et al., 2007), in each decision-making situation, subjects are confronted with the feasibility and desirability of different behavioral outcomes. The desirability refers to the valence of the result state of a certain behavior, and the feasibility refers to the easy or difficult degree of the process to achieve the result state. When the psychological distance is nearer, the individual forms a lower level of construal, and the attractiveness of the feasibility increases. When the psychological distance is farther, the individual forms a higher level of construal, and the attractiveness of the desirability is stronger. In ambiguity decision making, the feasibility of the risk option is higher than the ambiguity option because the probability of the lower limit of the ambiguity option is lower 
than the probability of the risk option and the attractiveness of options is derived from the aspect of feasibility, then the subjects are more inclined to choose the ambiguity option. The desirability of the risk option is higher than the ambiguity option because the attractiveness of options is derived from the aspect of desirability, the subjects are more inclined to choose the ambiguity option with higher desirability. In ambiguity decision making, when the psychological distance is longer, the construal level is higher and the desirability of options is more attractive, then the subjects are more inclined to choose the ambiguity option with higher desirability.

This study found that, no matter under which dimension of psychological distance, the influence of psychological distance on the ambiguity decision making possesses a similarity, which is consistent with the study results of Chen and $\mathrm{He}$ (2014). Chen and He (2014) thought that psychological distance can influence the decision selection by influencing the representation pattern of decision options (high or low construal level) and the weight distribution (weighted mode) of different option features. Because of the homogeneity among different types of psychological distances, they all have similar effects on the representation and weighting modes in the decision-making process, thereby creating similar influences on decision selection. In addition, Kim, Zhang, and Li (2008) also found that time distance and social distance have similar effects on consumer product evaluation and decision making. By investigating the similarity among the effects of time distance, space distance, social distance and probability on the ambiguity decision making, the paper further proves the equivalence among the effects of different dimensions of psychological distances on decision making.

\section{Conclusion and Prospect}

This study extends previous researches on the influence of psychological distance on ambiguity avoidance. Conclusions are drawn as follows: 1) There is a similarity among the effects of different dimensions of psychological distances on ambiguity decision making, and such dimensions include time distance, social distance, probability and spatial distance. 2) The closer the psychological distance is in decision-making, the lower the level of construal for decision events or objects, thereby bringing a bias towards ambiguity avoidance in the decision-making. However, this study only explored the influence of psychological distance on ambiguity avoidance in the context of gain. In our daily life, we should not only face the situation of gain, but also face the situation of loss. Therefore, future research should combine the gain and loss situation to explore the influence of psychological distance on ambiguity avoidance.

\section{References}

Camerer, Colin, F., Weber, \& Martin (1991). Recent Developments in Modelling Preferences: Uncertainty and Ambiguity. Journal of Risk \& Uncertainty, 5, 325-370.

Cen, Y. (2016). The Effect of Optimistic Bias: A Construal Level Perspective. Journal of Psychological Science. 
Chen, H. X., \& Hu, G. B. (2014). The Influence of Psychological Distance on Intertemporal Choice and Risk Selection. Acta Psychologica Sinica, 46, 677-690. https://doi.org/10.3724/SP.J.1041.2014.00677

Ellsberg, D. (1961). Risk, Ambiguity, and the Savage Axioms. Quarterly Journal of Economics, 75, 643-669. https://doi.org/10.2307/1884324

Fox, C. R., \& Weber, M. (2002). Ambiguity Aversion, Comparative Ignorance, and Decision Context. Organizational Behavior \& Human Decision Processes, 88, 476-498. https://doi.org/10.1006/obhd.2001.2990

Gong, H., Iliev, R., \& Sachdeva, S. (2012). Consequences Are Far Away: Psychological Distance Affects Modes of Moral Decision Making. Cognition. https://doi.org/10.1016/j.cognition.2012.09.005

Heath, C., \& Tversky, A. (1991). Preference and Belief: Ambiguity and Competence in Choice under Uncertainty. Journal of Risk \& Uncertainty, 4, 5-28. https://doi.org/10.1007/BF00057884

Kim, K., Zhang, M., \& Li, X. (2008). Effects of Temporal and Social Distance on Consumer Evaluations. Journal of Consumer Research, 35, 706-713. https://doi.org/10.1086/592131

Liberman, N., \& Trope, Y. (1998). The Role of Feasibility and Desirability Considerations in Near and Distant Future Decisions: A Test of Temporal Construal Theory. Journal of Personality \& Social Psychology, 75, 5-18. https://doi.org/10.1037/0022-3514.75.1.5

Liu, Y., \& Onculer, A. (2017). Ambiguity Attitudes over Time. Journal of Behavioral Decision Making, 30, 80-88. https://doi.org/10.1002/bdm.1922

Osmont, A., Cassotti, M., Agogué, M., Houdé, O., \& Moutier, S. (2015). Does ambiguity Aversion Influence the Framing Effect during Decision Making? Psychonomic Bulletin \& Review, 22, 572-577.

Pulford, B. D. (2009). Is Luck on My Side? Optimism, Pessimism, and Ambiguity Aversion. Quarterly Journal of Experimental Psychology, 62, 1079-1087. https://doi.org/10.1080/17470210802592113

Rubaltelli, E., Rumiati, R., \& Slovic, P. (2010). Do Ambiguity Avoidance and the Comparative Ignorance Hypothesis Depend on People's Affective Reactions? Journal of Risk and Uncertainty, 40, 243-254. https://doi.org/10.1007/s11166-010-9091-z

Todorov, A., Goren, A., \& Trope, Y. (2007). Probability as a Psychological Distance: Construal and Preferences. Journal of Experimental Social Psychology, 43, 473-482. https://doi.org/10.1016/j.jesp.2006.04.002

Trope, Y., \& Liberman, N. (2010). Construal-Level Theory of Psychological Distance. Psychological Review, 117, 440-463. https://doi.org/10.1037/a0018963 